\title{
3D Inverted Index with Cache Sharing for Web Search Engines
}

\author{
Esteban Feuerstein ${ }^{1}$, Veronica Gil-Costa ${ }^{2,5}$, Mauricio Marin ${ }^{4,5}$, \\ Gabriel Tolosa ${ }^{3,1}$, and Ricardo Baeza-Yates ${ }^{5}$ \\ 1 Universidad Nacional de Buenos Aires, Argentina \\ 2 Universidad Nacional de San Luis, Argentina \\ 3 Universidad Nacional de Lujan, Argentina \\ 4 Universidad de Santiago de Chile \\ 5 Yahoo! Labs Santiago, Chile
}

\begin{abstract}
Web search engines achieve efficient performance by partitioning and replicating the indexing data structure used to support query processing. Current practice simply partitions and replicates the text collection on the set of cluster processors and then constructs in each processor an index data structure. This paper proposes a different approach by constructing an index data structure that properly considers the fact that data is partitioned and replicated. This leads to a so-called $3 \mathrm{D}$ indexing strategy that outperforms current approaches. Performance is further boosted by introducing an application caching scheme devised to hold most frequently issued queries.
\end{abstract}

\section{Introduction}

The importance of text retrieval systems for the Web has grown dramatically during recent years due to the very rapid increase of available storage capacity. To ease the search of information, Web search engines (WSEs) are systems that index a portion of documents from the whole Web and allow to locate information through the formulation of queries. Well-known WSEs use the inverted index (or inverted file) 1 to speed up determination of relevant documents for queries.

The inverted index 110 is composed of a vocabulary table (which contains the $V$ distinct relevant terms found in the document collection) and a set of posting lists. The posting list for term $c \in V$ stores the identifiers of the documents that contain the term $c$, along with additional data used for ranking purposes. To solve a query, one must fetch the posting lists for the query terms, compute the intersection among them, and then compute the ranking of the resulting intersection set using algorithms like BM25 or WAND 2]. Hence, the precomputed data stored in the inverted index enables the very fast computation of the top-k relevant documents for a query.

Usually these systems are expected to hold the whole index in the distributed main memory held by the processors. There are two basic types of organization for distributed inverted indexes, namely document-based partition and termbased partitions (or local and global indexes respectively). In the former, documents are evenly distributed onto $P$ processors and an independent inverted

C. Kaklamanis et al. (Eds.): Euro-Par 2012, LNCS 7484, pp. 272-284, 2012.

(C) Springer-Verlag Berlin Heidelberg 2012 
index is constructed for each of the $P$ sets of documents. Therefore answering a conjunctive query requires computing at each processor the intersection of the terms that form the query, obtaining partial results by performing the ranking to select the top- $k$ local documents and then merging all those results.

In the term-partitioned index, a single inverted file is constructed from the whole document collection to then distribute evenly the terms with their respective posting lists onto the processors. In that way, to answer a conjunctive query one needs to determine which processor(s) hold the posting lists of the involved terms, then gather those lists in one processor and compute their intersection. Afterwards the ranking is performed over the resulting intersection set.

In our previous work [5], a 2-dimensional (2D) index was introduced. The 2D index combines document- and term-partitioning to get the "best of two worlds", i.e. to exploit the trade-off between the overhead due to the involvement of all processors in each query process as in the former, and the high communication costs required by the latter. In this paper we propose to extend the $2 \mathrm{D}$ index by adding processor replication, which has been widely used in conjunction with the document- and term-based approaches to improve throughput and fault tolerance [7.

We investigate the characteristics, performance and scalability of our extended $2 \mathrm{D}$ index called 3D index through the application of a performance evaluation framework (already used in [4]) that combines the usage of input query logs, average-cost analysis and stochastic values to compute realistic predictions of performance. The framework, based on the bulk-synchronous model of parallel computing (BSP) [12, blends empiric analysis and theoretical tools to predict the cost of real system executions which, otherwise, would be impossible to obtain in practice given the large scale of the cluster resources that would be required for experimentation.

We also improve the 3D index by adding a cache-sharing mechanism to save communication and computation overhead at the expense of an extra cost required to synchronize more processors. This new caching mechanism is also usable in simpler architectures as for example a term-partitioned index supporting replicas. The new caching mechanism constitutes an improvement to what has been proposed by Moffat et al. in [10].

The reminder of this paper is organized as follows. Section 2 presents the basic ideas behind the 3D distributed index. In Section 3 we introduce our cost estimation framework. In Section 4 we present the experimental setting. Sections 5 and 6] are devoted to show details and results of our experiments. Sections 7 and 8 respectively present related work and conclusions.

\section{3D Index}

The architecture we are proposing consists of the arrangement of a set of $P$ processors in a $3 \mathrm{D}$ cube, formed by replicating $D$ times each processor of a basic 2-dimensional matrix of $R$ rows and $C$ columns. On the row dimension, we apply the term-partition approach. On the column dimension we apply a documentpartition approach. In other words, the document collection is divided into $C$ 
sub-collections, each of which is allocated into a "column" of $R$ processors. Each processor holding a sub-collection $C_{i}$ with terms belonging to $R_{j}$ is replicated $D$ times (See Figure 1).

The $2 \mathrm{D}$ index can be replicated in different ways. Each one may imply different search algorithms, sharing resources policies, synchronization and fault tolerance approaches, among other features. For example, by simply replicating $D$ times the 2D index may improve the throughput roughly in a linear way as a function of the number of replicas, because the $D$ sets of processors form $D$ independent $2 \mathrm{D}$ index. Then each single $2 \mathrm{D}$ index replica processes a different set of queries. No communication is required between replicas, only among processors within the same replica. But it has some important drawbacks. The first one regards fault tolerance: the failure of a processor would imply the unavailability of a whole $2 \mathrm{D}$ index portion for a given set of queries.

The second issue regards the workload and may be explained as follows. For a fixed document collection and processor capacity, there is a minimum number of processors needed to handle the whole index on main memory. By simply maximizing the number of replicas would keep all the processors RAMs with the same load. No free memory is available for storing other cache structures. Meanwhile reducing the number of replicas and expanding the index over the $C$ and $R$ dimensions would allow to free RAM space in the processors that could be used for caching purposes.

A second approach consists on replicating $D$ times each individual processor holding a portion of the $2 \mathrm{D}$ index. In this case queries are solved by processors of different replicas. Therefore there is communication among replicas. This approach may allow a more flexible replacement policy in case of a failure of a processor. It also allows to share the caches memories among the processors in a more intelligent way at the expense of additional synchronization costs. We explore the trade-off between these costs in Section 6.

In Figure 1 we present a schematic view of our architecture and the query flow. We propose a setting in which all the processors have a dual role of query brokers and search nodes. Each query will be introduced to the system through one of

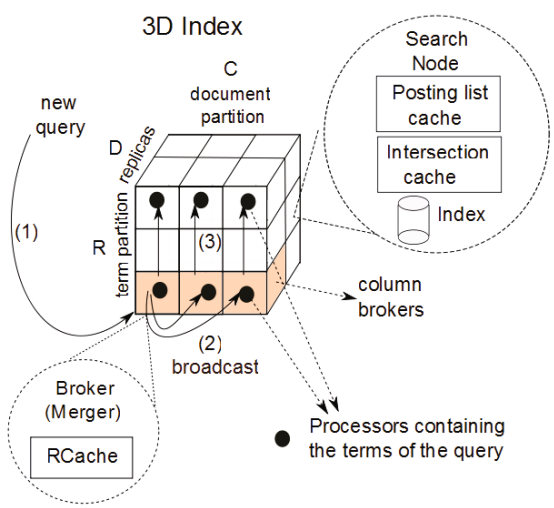

Fig. 1. 3D Index architecture 
the processors that will act as its broker. That processor will then distribute the query by sending it to a random processor of each column of the same replica, the column brokers (step 2 in Figure 1).

In each column the terms may be co-resident at the same processor (row) or not. Each column broker determines which rows contain the terms involved in the query, and sends the query to the processors in each of those rows (in that same replica). Afterwards, they perform the intersection and ranking of the posting list. Each column broker that has computed and ranked the intersection sends its results to the broker. The broker merges the partial results.

As a broker, each processor keeps a cache called RCache [6]. The RCache keeps the answers for frequent queries (they are query results composed of document IDs). Each processor, as a search node, keeps the inverted index in secondary memory. Also each processor holds a posting lists cache for most frequent terms and an intersection cache for previously computed intersections. The latter will be used as a resource shared by a subset of the processors using the approaches explored in Section 6 .

\section{Cost Estimation Methodology}

Our cost estimation framework is based on the bulk-synchronous model of parallel computing (BSP) [12, where computation is organized as a sequence of supersteps. In each of superstep the processors may perform computations on local data and/or send messages to other processors. The messages are available for processing at their destinations by the next superstep, and each superstep ends with the synchronization of the processors.

The total running cost of a BSP program is the cumulative sum of the costs of its supersteps, and the cost of each superstep is the sum of three components: computation, communication and synchronization. Computation cost is given by the maximum computation cost of a processor in the superstep, including also disk accesses. Communication cost is given by the maximum number of wordlength messages sent or received by a processor during the superstep multiplied by the cost of communicating one word.

Besides, we note that just for participating in the processing of a query a processor incurs in certain overheads which must also be taken into consideration such as disk access, thread scheduling, etc. We model computation, communication and disk access overheads separately. We also separate computation cost taking into account partial costs of performing intersection, ranking and merging operations. Therefore, the total cost of a superstep is defined as:

where

$$
\max _{p}(\max (\text { computation }+ \text { communication }), \text { disk })+\text { Sync }
$$

- $p$ ranges over all participating processors

- computation $=$ processing overhead + intersection cost + ranking cost + merge cost

- communication $=$ communication overhead + communication cost

- disk $=$ disk overhead + disk access cost 
The overheads of computation, communication and disk costs of formula 1 are computed as the total number of operations (of each kind) performed in the processor. These values are then multiplied by appropriate constants corresponding to each operation. All these constants were obtained through benchmark programs run on actual hardware. Table 1 summarizes the primitive components (computation and communication costs) assigned to each query.

In our context, communication cost is by far smaller than computational cost since the sum of costly operations requiered to solve queries is much larger than the cost of sending small messages of size $|q|$ or size $K$ among processors. Selecting the top-k results for a query requires operating on posting lists of size $x \gg K$.

Table 1. Primitive operations and concepts used in the simulation, and their values

\begin{tabular}{|c|c|c|}
\hline Notation & Meaning & Cost \\
\hline$t_{i}(x, y)$ & $\begin{array}{l}\text { Time required to compute the intersection } \\
\text { of two lists of lengths } x \text { and } y \text {. }\end{array}$ & $\begin{array}{l}t_{i}(x, y)= \\
\min (x \log y, x+y) / 6\end{array}$ \\
\hline$t_{m}(x)$ & $\begin{array}{l}\text { Time required to merge a set of lists of to- } \\
\text { tal length } x \text {. }\end{array}$ & $x / 6$ \\
\hline$t_{r}(x)$ & $\begin{array}{l}\text { Time to select the top-k results in a list of } \\
x \text { items. }\end{array}$ & $x$ \\
\hline$\overline{I(x, y)}$ & $\begin{array}{l}\text { Intersection length of two lists of length } \\
x \leq y \text { and constant values } s \text { and } a \text {. }\end{array}$ & $\begin{array}{l}s * x * y / 10^{4}+(s+a) * x \\
\text { according to } 4\end{array}$ \\
\hline processing overhead & $\begin{array}{l}\text { Overhead due to the participation of a pro- } \\
\text { cessor in a query. }\end{array}$ & 0.1 nanoseconds \\
\hline processing cost & Variable processing cost. & 1 nanosecond \\
\hline $\begin{array}{l}\text { communication over- } \\
\text { head }\end{array}$ & $\begin{array}{l}\text { Overhead for transmitting any number of } \\
\text { bytes }\end{array}$ & 50000 nanoseconds \\
\hline communication cost & Variable communication cost & 50 nanoseconds (5) \\
\hline disk overhead & Fixed cost for accessing disk & 700 nanoseconds \\
\hline disk access & Variable disk cost (per byte) & 0.4 nanoseconds $(0.2)$ \\
\hline
\end{tabular}

\section{Experimental Setting}

In this section we describe the general framework used for our experiments. We estimated the cost of different $3 \mathrm{D}$ index configuration by combining the variables $P, C, R$ and $D$, where $P$ is the total number of processors. We run 300,000 queries with $P$ ranging from 32 to 2048. The number of replicas ranges from $D=2$ to 64 , and the number of columns and rows range from 1 to $P / 2$ with $P=C \times R \times D$.

Queries were selected from a query log with $36,389,567$ queries. We preprocessed the query log following the rules applied in [6] by removing stopwords and completely removing any query consisting only of stopwords. The resulting query trace has $16,900,873$ queries. Then, we randomly selected about one million queries where $42 \%$ are unique queries, $14 \%$ occur twice and $6 \%$ occur three times. As expected, the queries' lengths are not uniformly distributed. The relative frequencies of one, two, three and four-term queries is respectively $24 \%$, 
$33 \%, 23 \%$ and $11 \%$. The basic index and the posting lists were built using a 1.5TB sample of the UK's web obtained from Yahoo! Search engine.

To set the values of the different primitive operation costs and constants required by our cost estimation framework, we used the same results of the benchmark programs reported in 44. The values are expressed relative to a baseline in terms of ranking time defined as $t_{r}(x)=x$ (See Table 1).

To determine the communication and disk access costs we run specific benchmark programs. In both cases we used standard available technology (serial ATA disks, and a Gigabit Ethernet). Hence, both estimations can be seen as lower bounds for the real performance on a specialized and fine-tuned architecture. Benchmark programs where run on a Linux operating system with MPI.

To compute disk access time we considered the positioning of the read/write head (lseek) in response to a sequence of a random sequence of positioning depending on the length of the stream of bytes, and the time employed to read a block. We used blocks of different sizes (1 to 3000 bytes). We run six different tests combining reset and update operations. The results show that the cost of accessing $x$ bytes can be approximated with the function $0.4 x+700$ nanoseconds.

We run benchmark programs to obtain the communication time between pairs of processors with different message sizes (in a range of 1-3000 bytes). We considered messages in the unicast and broadcast modes. We observed that the communication cost grows with the number of transmitted bytes. This cost can be approximated with the expression $50 x+50000$ nanoseconds. From these benchmark programs we established the values reported in Table 1.

\section{Evaluation of the 3D Index}

In this section we report the experiments performed to analyze the efficiency of our 3D index. We use a basic architecture as described in Section 2. All queries are solved within one replica of the $2 \mathrm{D}$ index, so no synchronization is needed among all processors at the end of every superstep, but just among those participating in the same replica. Figure 2 at left shows this approach. The black ball represents processors involved in the query process. No communication between processors of different replicas is required.

The size of cache memories used in the following experiments is reported in Section 6. We also show cost normalized to 1 to better illustrate the difference between strategies. In all cases we divide the values by the observed maximum in the respective experiment.

Figure 3 at left shows that if we allow replicas to proceed without synchronization, the best choice is to have the maximum possible number of replicas. That is natural as the whole stream of queries can be divided evenly among all replicas. In this way we obtain an optimal speed-up.

Figure 3at right shows in the $y$-axis the optimal number of rows and columns selected for different values of replicas $D$ in the $x$-axis. Namely, the configurations $R \times C$ with the minimum estimated cost. We observed that those configurations with many replicas (where each replica is as small as possible) it is 


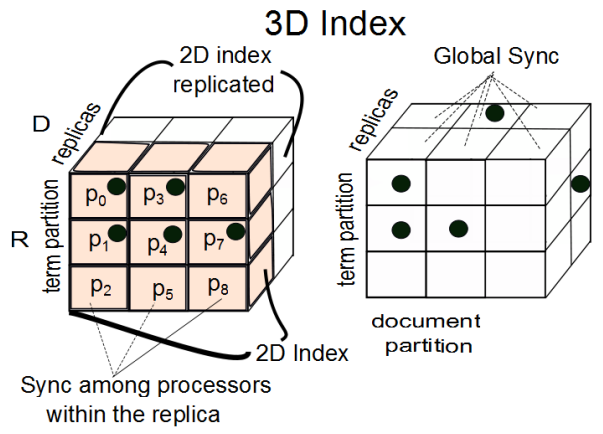

Fig. 2. Left: queries solved within a single 2D replica. Right: queries solved among all processors.

better to arrange the processors with $R=1$ and $C$ columns, i.e. a local index. However, configurations with a small $D$ (and hence bigger $R \times C$ ) present the typical $2 \mathrm{D}$ index trade-off between communication and overhead costs presented among the term-partition and document-partition indexes.
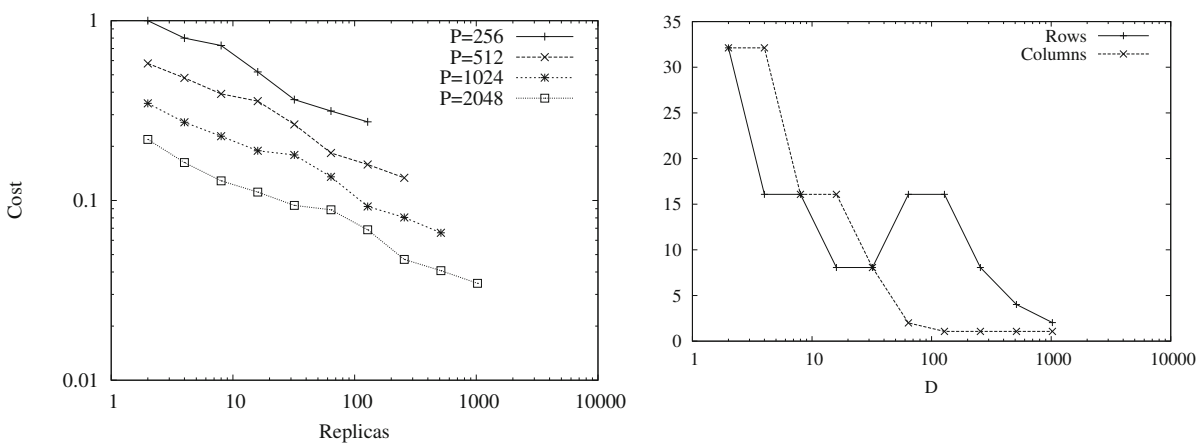

Fig. 3. Non synchronization among replicas. Left: Estimated cost. Right: Optimal number of columns $C$ and rows $R$ as a function of $D$ with $P=2048$.

Figure 4 at left shows results forcing synchronization among all $P$ processors at the end of each superstep. In this approach queries are solved using processors belonging to different replicas. For a given query, replicas are selected in a roundrobin fashion. As communication is performed among all processors a global synchronization is required. Figure 2 at right shows how a query $q$ represented as a black ball is processed by processors belonging to different replicas.

Figure 4 at left shows the estimated cost as a function of the number of columns. The optimal value of $D$ grows with $P$, but is far from tending to $D=P$. Moreover, the natural trade-off between the benefits of the global and local indexes makes that the optimal configuration can be found using a pure 2D configuration, i.e. using between 8 and 16 columns. 

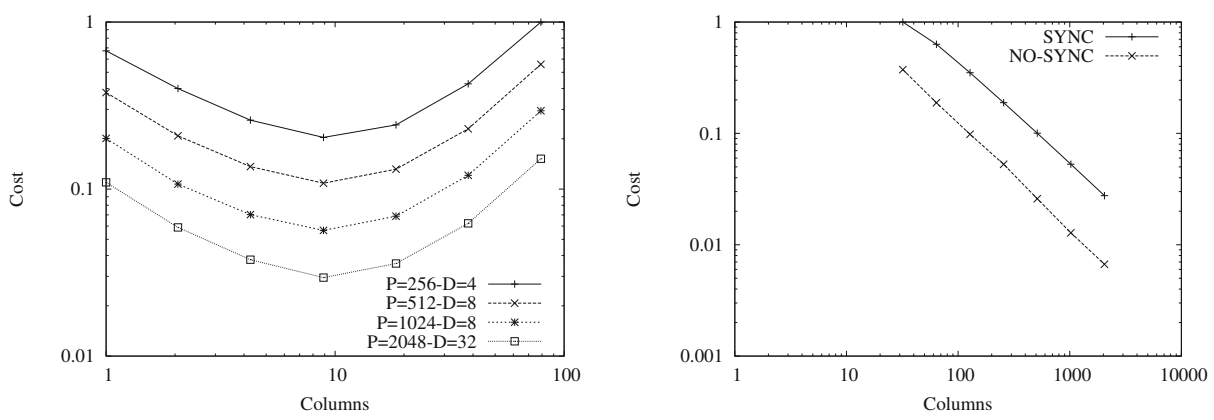

Fig. 4. Left: All processors are synchronized at the end of every superstep. Right: replica synchronization vs. global synchronization.

Figure 4 at right compares the estimated cost for different values of $P$ when synchronization is performed only among processors within a single replica $(\mathrm{NO}$ $S Y N C$ ) versus global synchronization $(S Y N C)$ involving all processors. With few processors, synchronizing only processors within a replica significantly reduces the total cost. But with $P \geq 500$, the difference between both approaches is less than 1\%. Moreover, the SYNC approach does not present the same faulttolerance and workload drawbacks as described in Section 2 . We conclude that, without any cache sharing mechanism (as the one introduced in Section 6), the number of replicas must be as big as possible.

\subsection{Scalability of the 3D Index}

In this section we study the scalability of the $3 \mathrm{D}$ index as the size of the document collection increases. We estimate the total cost of computing 300,000 queries for $P=512,1024$ and 2048. We selected the best configurations for each $P$. Namely, the combination $P=R \times C \times D$ which minimizes the total cost. We used $R \times C \times D$ defined by: $R=[2 \ldots 64], C=[4 \ldots 64]$ and $D=[4 \ldots 16]$. The rest of the parameters were kept unchanged, as described in Section 4 . We force the synchronization of all the processors.

Figure 5] at left shows the estimated cost in the $y$-axis. The $x$-axis values represent the growth factor of posting lists. The main conclusion of this experiment is that, for a fixed value of $P$, the estimated cost of the system grows proportionally to the logarithm of the size of the document collection. This behavior is expected due to the fact that the running time of list intersections is logarithmic. Interestingly Figure 5at right shows that the speed-up is constant as we increase the number of processors. Sometimes it even tends to improve as the collection size grows. 

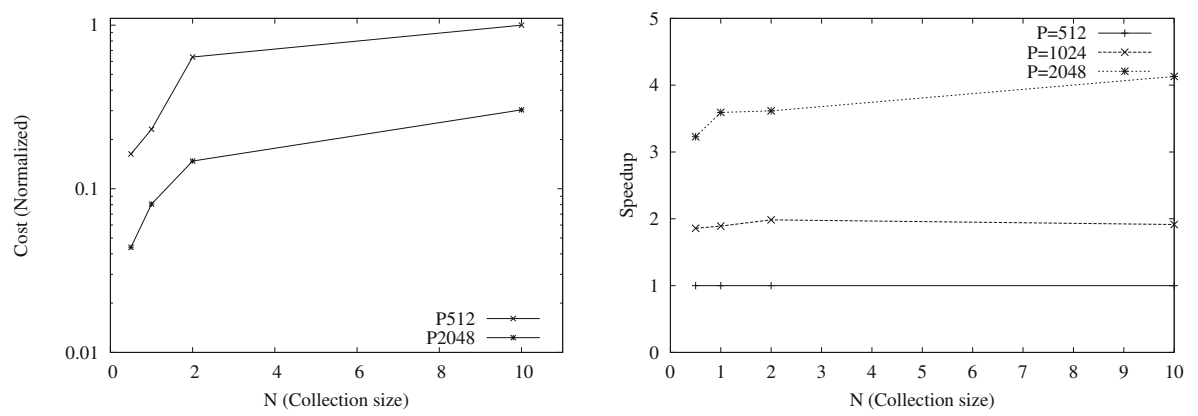

Fig. 5. Left: Estimated cost as the collection size varies, for different number of processors. Right:Speed-up as the collection size varies.

\section{Pipelined Caching}

In this section we propose a novel idea for managing the individual RAM memories of the processors as part of a big common cache. We propose to use a larger cache shared among processors. A different idea with the same goal was presented in [4] for a simpler index (local index with replication). The results in [4] showed that improvements in the performance can be obtained with some extra communication cost. We apply this idea to the intersection cache.

Our schema is applied to the set of processors forming a "column slice" of our 3D cube. Each column slice is a term-partitioned index with replicas for a particular sub-collection of documents (see Fig 6(a) (c)]. Within each slice the processors are partitioned in subsets (called teams). We use a hash function $H$ to map each query term to one processor of the team. Different ways of constructing the teams determine different algorithms. We will explore three particular cases:

(a) One Row-many Replicas teams: Each slice is partitioned by terms in $R$ teams. Each team has $D$ replicas (Figure 6(a)).

(b) One Replica - many Rows teams: Each slice is divided in $D$ teams. Each team has $R$ processors. (Figure 6(b) .

(c) Slice-teams: All the processors in each slice form one single team (Figure 6(c)].

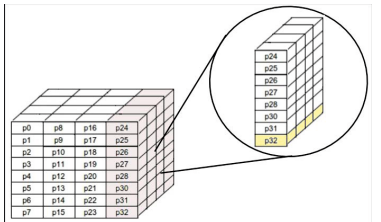

(a) One Row-Many Repli- (b) One Replica - Many cas teams

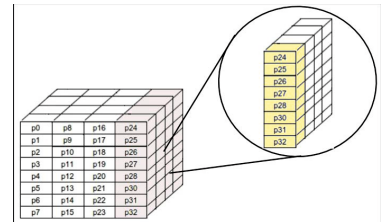

Rows teams

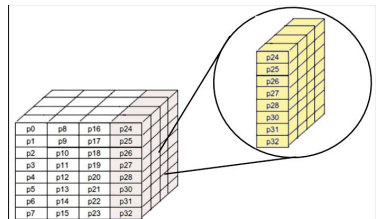

(c) Slice teams

Fig. 6. Pipelined caching 
Algorithm 1 explains our caching policy. We explain the query process inside one team. Upon request of a query $t_{1} \wedge t_{2} \ldots \wedge t_{m}$, the broker directs the query to one random processor in each column of its replica. Each of these $C$ processors will be referred to as a column-broker. The column-broker analyzes the query and interacts with the processors of its team to evaluate if there are, in the team's collective cache, any previously cached intersections that may be useful to compute the answer. What differentiates the three approaches presented above is the construction of the hashing function $H$, whose co-domain is always the team of the column-broker. For any set of terms $\left\{t_{1} \ldots t_{i}\right\}, p_{H\left(t_{1} \ldots t_{i}\right)}$ denotes the processor that, according to the hashing function $H$, could have in its cache the result of the intersection $t_{1} \wedge t_{2} \ldots \wedge t_{i}$.

The column-broker first looks for the whole or part of the query intersection (lines 1-4). If some intersection is found, the cached result is requested to the processor that holds it (lines 5-8). Otherwise an empty list is set (lines 9-11). This list $\ell$ will contain the processors identifiers that hold the remaining terms of the query (lines 13-16). If the whole query is found in cache, the retrieved list is returned (line 17). Each processor that computes an intersection caches the result and sends it to the following processor in $\ell$. We use $O(t)$ to denote the processor that holds term $t$ in any replica.

For example, for a four-term query $t_{1} \wedge t_{2} \wedge t_{3} \wedge t_{4}$ the column-broker in each column looks for the intersection $t_{1} \wedge t_{2} \wedge t_{3} \wedge t_{4}$ in a particular processor of the team $\left(p_{H\left(t_{1} \ldots t_{4}\right)}\right)$. If that fails, in a second step, it looks for $t_{1} \wedge t_{2} \wedge t_{3}$ on the corresponding processor $\left(p_{H\left(t_{1} \ldots t_{3}\right)}\right)$ and so on. When a hit is found the partial result of the intersection is retrieved from cache, and a schedule in $\ell$ is prepared to complete (by computation) the remaining intersections.

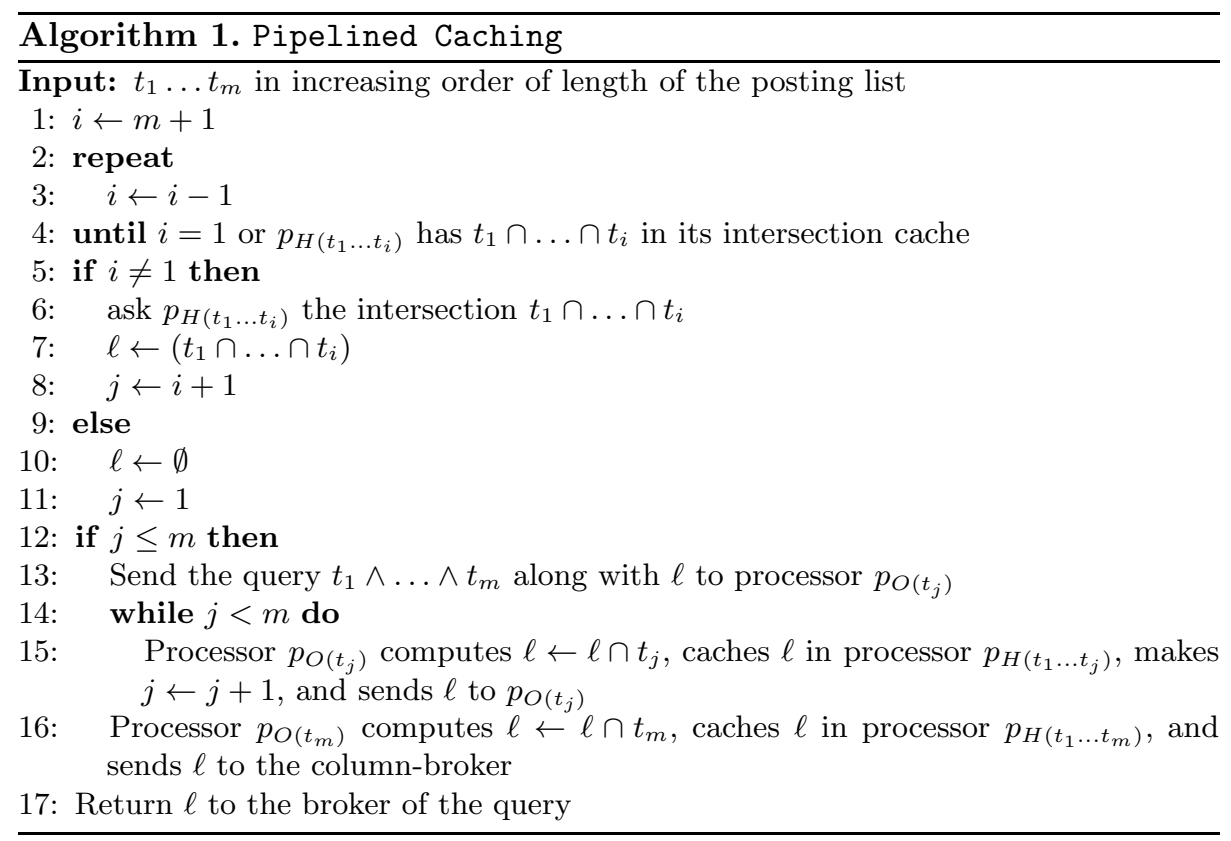




\subsection{Results}

In the following experiments we set the size of the intersection cache of each processor in terms of the number of "entries", i.e. a certain number of pre-computed intersections. We set a fixed size of $100 K$ cache entries for each processor so the total size of the intersection cache is $100 K \times P$ entries. This enables a natural "growing" property when adding processors to the whole system. The total space of the intersections cache in a particular team varies according to the combinations of $R \times C \times D$ and the chosen scheme. With the One Row-Many Replicas, the total size of the cache of each team is $100 K \times D$. For One Replica -Many Rows it is $100 K \times R$ and finally for the Slice scheme we have $100 K \times R \times D$.

The RCache implemented at the broker side uses a SDC policy [3]. We made a "warm up" of the RCache with $200 K$ query results. Finally, all the processors maintain caches of posting lists to reduce secondary memory access. This cache is administered with the standard LRU strategy.

In the baseline approach (2D approach) each processor cache is used and managed locally. Any information about previously computed intersections of posting lists is local to the owner of the cache memory.

Figure 7 at left shows the improvement obtained by the cache sharing model One Replica - Many Rows team. With $P=512$, this new approach reduces the estimated cost by $40 \%$. With the Slice approach the improvement is up to $55 \%$.
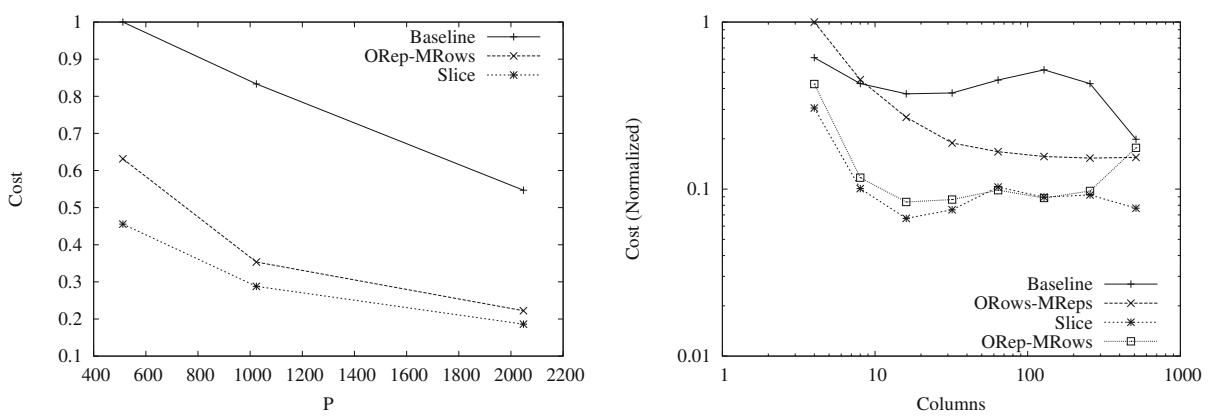

Fig. 7. Left: Normalized costs of the caching strategies. Right: Estimated cost as a function of the number of columns.

Figure 7 at right shows the estimated cost of the three cache sharing strategies and the baseline approach as a function of the number of columns for the best $D$ value. Again the Slice approach presents very good results followed by the One Replica -Many Rows approach. The Slice allows an efficient use of the cache space since it allows to maximize the memory space shared between nodes of different replicas. 


\section{Related Work}

The 2D index that combines document- and term-partitioning was originally proposed in [5]. Other variants of these basic schemes have been proposed in 1113. In this work, we use a cost estimation framework [4] based on BSP [12. Recently the BSP model has been widely adopted by systems used in off-line computations for search engines (e.g., index construction) such as Google's Pregel 8]. In 4] the cost estimation framework was used for a simpler index (local index with replication) showing performance improvements due to the reduction in computation time and disk access. Moreover, the caches were shared by the set of replicas of each partition (in that work, a column). Within each partition all the computation was centralized at the broker of the query, which asked all its "replicas" for possible intermediate results in their caches. No "query routing" was considered there.

Also, our approach is related to the pipelined query-evaluation methodology of [10] in which partially evaluated queries are passed amongst the set of processors that host the query terms. In other words, it constructs a path or pipeline schedule through which the query "flows" to be solved. But in our case the path depends on the content of the shared cache. We combine this pipelined approach with an intersection cache distributed across the replicas of processors. The initial architecture of [10] suffered from load imbalance across the nodes of the cluster. That problem is partially solved in 9] through replication of popular terms in different processors.

\section{Conclusions}

In this work we have presented a 3D inveted index along a new caching algorithm for Web search engines. Replication is used as a mean to increase query throughput and to support failures. The 3D index efficiently handles replication and outperforms the 2D index which in turn outperforms well-known distributed indexes [5]. The number of rows, columns and replicas can be chosen so as to optimize the overall performance. We explored two approaches to replicate the index: SYNC and NO-SYNC. There is a trade-off. The NO-SYNC presents better performance but is less fault tolerant and viceversa.

The proposed caching algorithm is designed to make an optimized use of the distributed memory for caching posting list intersections. This significantly reduces communication of posting lists among nodes (processors). Intersections of popular terms in queries tend to remain cached, so the load of the holders of those terms is also reduced. Moreover, the hashing function of the caching algorithm can be fine-tunned to optimize a combination of hit ratio and load balance of nodes. 


\section{References}

1. Baeza-Yates, R., Ribeiro-Neto, B.: Modern Information Retrieval - the concepts and technology behind search, 2nd edn. Pearson Education Ltd. (2011)

2. Broder, A.Z., Carmel, D., Herscovici, M., Soffer, A., Zien, J.Y.: Efficient query evaluation using a two-level retrieval process. In: CIKM, pp. 426-434 (2003)

3. Fagni, T., Perego, R., Silvestri, F., Orlando, S.: Boosting the performance of web search engines: Caching and prefetching query results by exploiting historical usage data. ACM Trans. Inf. Syst. 24, 51-78 (2006)

4. Feuerstein, E., Gil-Costa, V., Mizrahi, M., Marin, M.: Performance Evaluation of Improved Web Search Algorithms. In: Palma, J.M.L.M., Daydé, M., Marques, O., Lopes, J.C. (eds.) VECPAR 2010. LNCS, vol. 6449, pp. 236-250. Springer, Heidelberg (2011)

5. Feuerstein, E., Marin, M., Mizrahi, M., Gil-Costa, V., Baeza-Yates, R.: TwoDimensional Distributed Inverted Files. In: Karlgren, J., Tarhio, J., Hyyrö, H. (eds.) SPIRE 2009. LNCS, vol. 5721, pp. 206-213. Springer, Heidelberg (2009)

6. Gan, Q., Suel, T.: Improved techniques for result caching in web search engines. In: WWW, pp. 431-440 (2009)

7. Gomez-Pantoja, C., Marin, M., Gil-Costa, V., Bonacic, C.: An Evaluation of FaultTolerant Query Processing for Web Search Engines. In: Jeannot, E., Namyst, R., Roman, J. (eds.) Euro-Par 2011, Part I. LNCS, vol. 6852, pp. 393-404. Springer, Heidelberg (2011)

8. Malewicz, G., Austern, M.H., Bik, A.J., Dehnert, J.C., Horn, I., Leiser, N., Czajkowski, G.: Pregel: a system for large-scale graph processing. In: PODC, p. 6 (2009)

9. Moffat, A., Webber, W., Zobel, J.: Load balancing for term-distributed parallel retrieval. In: SIGIR, pp. 348-355 (2006)

10. Moffat, A., Webber, W., Zobel, J., Baeza-Yates, R.: A pipelined architecture for distributed text query evaluation. Information Retrieval 10, 205-231 (2007)

11. Tang, C., Dwarkadas, S.: Hybrid global-local indexing for efficient peer-to-peer information retrieval. In: NSDI, p. 16 (2004)

12. Valiant, L.G.: A bridging model for multi-core computing. J. Comput. Syst. Sci. 77(1), 154-166 (2011)

13. Xi, W., Sornil, O., Luo, M., Fox, E.A.: Hybrid Partition Inverted Files: Experimental Validation. In: Agosti, M., Thanos, C. (eds.) ECDL 2002. LNCS, vol. 2458, pp. 422-431. Springer, Heidelberg (2002) 